\title{
Planck Quantization of Newton and Einstein Gravitation
}

\author{
Espen Gaarder Haug \\ Norwegian University of Life Sciences, Campus Ås, Norway \\ Email: espenhaug@mac.com
}

Received 3 March 2016; accepted 20 June 2016; published 23 June 2016

Copyright (C) 2016 by author and Scientific Research Publishing Inc.

This work is licensed under the Creative Commons Attribution International License (CC BY).

http://creativecommons.org/licenses/by/4.0/

(c) (i) Open Access

\begin{abstract}
In this paper we rewrite the gravitational constant based on its relationship with the Planck length and based on this, we rewrite the Planck mass in a slightly different form (that gives exactly the same value). In this way we are able to quantize a series of end results in Newton and Einstein's gravitation theories. The formulas will still give exactly the same values as before, but everything related to gravity will then come in quanta. This also gives some new insight; for example, the gravitational deflection of light can be written as only a function of the radius and the Planck length. Numerically this only has implications at the quantum scale; for macro objects the discrete steps are so tiny that they are close to impossible to notice. Hopefully this can give additional insight into how well or not so well (ad hoc) quantized Newton and Einstein's gravitation is potentially linked with the quantum world.
\end{abstract}

\section{Keywords}

Quantized Gravitation, Gravitational Constant, Escape Velocity, Gravitational Time Dilation, Schwarzschild Radius, Planck Length, Bending of Light, Planck Mass, Planck Length

\section{Foundation}

We suggest that Newton's gravitational constant [1] could be written as a function of Planck's reduced constant

$$
G_{p}=\frac{l_{p}^{2} c^{3}}{\hbar}
$$

where $\hbar$ is the reduced Planck's constant, $c$ is the well tested round-trip speed of light, and $l_{p}$ is the Planck length [2]. We could call this Planck's form of the gravitational constant. This way of writing Newton's gravitational constant does not change the value of the constant. If one knows the Planck length, then the 
gravitational constant is known, or alternatively and more practically one can calibrate the Planck length based on empirical measurements of the gravitational constant. There is still considerable uncertainty in the exact measurement of the gravitational constant. Experimentally, substantial progress has been made in recent years based on various methods. See, for example, [3]-[7]. Also the relationship between physical constants from the microcosms (subatomic world) and the macrocosms (cosmos) plays an important role in physics and a continuous effort is going into improving our measurements and understanding of these relationships. See, for example, [8].

As shown by Haug [9], the Planck form of the gravitational constant enables us to rewrite the Planck length as

$$
l_{p}=\sqrt{\frac{\hbar G_{p}}{c^{3}}}=\sqrt{\frac{\hbar \frac{l_{p}^{2} c^{3}}{\hbar}}{c^{3}}}=l_{p}
$$

and the Planck mass as

$$
m_{p}=\sqrt{\frac{\hbar c}{G_{p}}}=\sqrt{\frac{\frac{\hbar c}{l_{p}^{2} c^{3}}}{\hbar}}=\frac{\hbar}{l_{p}} \frac{1}{c}
$$

Using the gravitational constant in the Planck form, as well as the rewritten Planck units, we are easily able to modify a series of end results from Newton and Einstein's gravitational theories to contain quantization as well.

\section{Newton's Universal Gravitational Force}

Newton's gravitational force is given by

$$
F_{G}=G_{p} \frac{m_{1} m_{2}}{r^{2}}
$$

Using the gravitational constant of the form $G_{p}=\frac{l_{p}^{2} c^{3}}{\hbar}$ and the Planck mass of $m_{p}=\frac{\hbar}{l_{p}} \frac{1}{c}$, we can rewrite Newtons gravitational force for two Planck masses as

$$
\begin{gathered}
F_{G_{P}}=G_{p} \frac{m_{p} m_{p}}{r^{2}} \\
F_{G_{P}}=\frac{l_{p}^{2} c^{3}}{\hbar} \frac{\frac{\hbar}{l_{p}} \frac{1}{c} \frac{\hbar}{l_{p}} \frac{1}{c}}{r^{2}}=\frac{\hbar c}{r^{2}}
\end{gathered}
$$

In the special case where $r=l_{p}$, we get

$$
F_{G_{p}}=\frac{\hbar}{l_{p}} \frac{c}{l_{p}}
$$

It seems from this that gravity potentially could be related to hits per second, even if the output naturally is the same as from the standard formula. For large masses the form will be

$$
\begin{gathered}
F_{G_{p}}=G_{p} \frac{N_{1} m_{p} N_{2} m_{p}}{r^{2}} \\
F_{G_{p}}=G_{p} \frac{N_{1} N_{2} m_{p}^{2}}{r^{2}} \\
F_{G_{p}}=\frac{l_{p}^{2} c^{3}}{\hbar} \frac{N_{1} N_{2} \frac{\hbar^{2}}{l_{p}^{2}} \frac{1}{c^{2}}}{r^{2}}
\end{gathered}
$$




$$
F_{G_{p}}=N_{1} N_{2} \frac{\hbar}{r^{2}} c
$$

where $N_{1}$ is the number of Planck masses in object one and $N_{2}$ is the number of Planck masses in object two. In the case where the two masses are of equal size, we have

$$
F_{G_{P}}=N^{2} \frac{\hbar}{r^{2}} c
$$

\section{Escape Velocity at the Quantum Scale}

The traditional escape velocity [10]-[12] is given by

$$
v_{e}=\sqrt{\frac{2 G M}{r}}
$$

where $G$ is the traditional gravitational constant, $M$ is the mass of the object we are "trying" to escape from, and $r$ is the radius of that object. In other words, we stand at the surface of the object, for example a hydrogen atom or a planet. Based on the gravitational constant written in the Planck form, we can find the escape velocity at Planck scale; see the Appendix for a derivation from "scratch". It must be

$$
\begin{gathered}
v_{e, p}=\sqrt{\frac{2 G_{p} N m_{p}}{r}} \\
v_{e, p}=\sqrt{\frac{2 N \frac{l_{p}^{2} c^{3}}{\hbar} \frac{\hbar}{l_{p}} \frac{1}{c}}{r}} \\
v_{e, p}=\sqrt{\frac{2 N l_{p} c^{2}}{r}} \\
v_{e, p}=c \sqrt{\frac{2 N l_{p}}{r}}
\end{gathered}
$$

where $N$ is the number of Planck masses in the planet or mass in question.

A particularly interesting case is when we only have one Planck mass $N=1$ and $r=2 l_{p}$ (this is actually the Schwarzschild radius of a Planck particle). This gives us

$$
\begin{gathered}
v_{e, p}=c \sqrt{\frac{2 \times 1 \times l_{p}}{2 l_{p}}} \\
v_{e, p}=c
\end{gathered}
$$

as the escape velocity for a particle with Planck mass is $c$. Next we will see if the formula above can also be used to calculate the escape velocity of Earth. The Earth's mass is $5.972 \times 10^{24} \mathrm{~kg}$. We must convert this to the number of Planck masses. The Planck mass is

$$
m_{p}=\frac{\hbar}{l_{p}} \frac{1}{c} \approx 2.17651 \times 10^{-8}
$$

The Earth's mass in terms of the numbers of Planck masses must be $\frac{5.972 \times 10^{24}}{2.17651 \times 10^{-8}} \approx 2.74388 \times 10^{32}$. Further the radius of the Earth is $r \approx 6371000$ meters. We can now simply plug this into the Planck scale escape velocity:

$$
v_{e, p}=c \sqrt{\frac{2 N l_{p}}{r}}
$$




$$
v_{e, p}=299792458 \times \sqrt{\frac{2 \times 2.74388 \times 10^{32} \times 1.61622837 \times 10^{-35}}{6371000}} \approx 11185.7 \text { meters/second }
$$

which is equal to $40,269 \mathrm{~km} / \mathrm{h}$, the well-known escape velocity from the Earth's gravitational field. We think our new way of looking at gravity could have consequences for the understanding of gravity. Gravitation must come in discrete steps and the escape velocity must also come in discrete steps for a given radius; this is because the amount of matter likely comes in discrete steps.

\section{Orbital Speed}

The orbital speed is given by

$$
v_{o} \approx \sqrt{\frac{G M}{r}}
$$

We can rewrite this in the form of the Planck gravitational constant and the Planck mass as

$$
\begin{gathered}
v_{o, p} \approx \sqrt{\frac{G_{p} N m_{p}}{r}} \\
v_{o, p} \approx \sqrt{\frac{\frac{l_{p}^{2} c^{3}}{\hbar} N \frac{\hbar}{l_{p}} \frac{1}{c}}{r}} \\
v_{o, p} \approx c \sqrt{\frac{N l_{p}}{r}} .
\end{gathered}
$$

This can also be written as

$$
v_{o, p} \approx \frac{v_{e}}{\sqrt{2}}=c \sqrt{\frac{N l_{p}}{r}}
$$

\section{Gravitational Acceleration Field}

The gravitational acceleration field in modern physics is given by

$$
g=\frac{G M}{r^{2}}
$$

This can be rewritten in quantized form as

$$
\begin{gathered}
g=\frac{G_{p} M}{r^{2}} \\
g=\frac{\frac{l_{p}^{2} c^{3}}{\hbar} N \frac{\hbar}{l_{p}} \frac{1}{c}}{r^{2}} \\
g=\frac{N l_{p} c^{2}}{r^{2}}
\end{gathered}
$$

\section{Gravitational Parameter}

The standard gravitational parameter is given by

$$
\mu=G M
$$

This can be rewritten in quantized form as

$$
\mu_{p}=G_{p} M
$$




$$
\begin{gathered}
\mu_{p}=G_{p} N m_{p} \\
\mu_{p}=\frac{l_{p}^{2} c^{3}}{\hbar} N \frac{\hbar}{l_{p}} \frac{1}{c} \\
\mu_{p}=N l_{p} c^{2}
\end{gathered}
$$

\section{Kepler's Third Law of Motion}

The Newtonian “mechanics version” of Kepler's third law of motion for a circular orbit is given by

$$
\frac{P^{2}}{a^{3}}=\frac{4 \pi^{2}}{G\left(M_{s}+m\right)}
$$

where $M_{\mathrm{s}}$ is the mass of the Sun, $m$ the mass of the planet, $P$ is the period, and $a$ is the semi-major axis. This can be rewritten as

$$
\begin{gathered}
\frac{P^{2}}{a^{3}}=\frac{4 \pi^{2}}{G_{p}\left(N_{1} m_{p}+N_{2} m_{p}\right)} \\
\frac{P^{2}}{a^{3}}=\frac{4 \pi^{2}}{\frac{l_{p}^{2} c^{3}}{\hbar}\left(N_{1} \frac{\hbar}{l_{p}} \frac{1}{c}+N_{2} \frac{\hbar}{l_{p}} \frac{1}{c}\right)} \\
\frac{P^{2}}{a^{3}}=\frac{4 \pi^{2}}{l_{p} c^{2}\left(N_{1}+N_{2}\right)}
\end{gathered}
$$

where $N_{1}$ is the number of Planck masses in the mass of the Sun $M_{s}$ and $N_{2}$ is the number of Planck mass of the planet $m$. In the case where the planet's mass is much smaller than the Sun's mass, we can use the following approximation

$$
\frac{P^{2}}{a^{3}} \approx \frac{4 \pi^{2}}{l_{p} c^{2} N}
$$

where $N$ is now the number of Planck masses in the Sun.

\section{Gravitational Time Dilation at Planck Scale}

Einstein's gravitational time dilation [13] is given by

$$
t_{0}=t_{f} \sqrt{1-\frac{2 G M}{r c^{2}}}=t_{f} \sqrt{1-\frac{v_{e}^{2}}{c^{2}}}
$$

where $v_{e}$ is the traditional escape velocity. We can rewrite this in the form of quantized escape velocity (derived above).

$$
\begin{gathered}
t_{o}=t_{f} \sqrt{1-\frac{v_{e, p}^{2}}{c^{2}}} \\
t_{o}=t_{f} \sqrt{1-\frac{\left(c \sqrt{2 N \frac{l_{p}}{r}}\right)^{2}}{c^{2}}} \\
t_{o}=t_{f} \sqrt{1-\frac{2 N l_{p}}{r}}
\end{gathered}
$$


Let's see if we can calculate the time dilation at, for example, the surface of the Earth from Planck scale gravitational time dilation. The Earth's mass is $5.972 \times 10^{24} \mathrm{~kg}$. And again, the Earth's mass in terms of the Planck mass must be $\frac{5.972 \times 10^{24}}{2.17651 \times 10^{-8}} \approx 2.74388 \times 10^{32}$. Further, the radius of the Earth is $r \approx 6371000$ meters. We can now just plug this into the quantized gravitational time dilation

$$
\begin{aligned}
& t_{o}=t_{f} \sqrt{1-\frac{2 N l_{p}}{r}} \\
& t_{o}=t_{f} \sqrt{1-\frac{2 \times 2.74388 \times 10^{32} \times 1.61622837 \times 10^{-35}}{6371000}} \approx t_{f} \times 0.999999999303915
\end{aligned}
$$

That is for every second that goes by in outer space (a clock far away from the massive object), 0.99999999930391500 seconds goes by on the surface of the Earth. That is to say, for every year in outer space (very far from the Earth), there are about 22 milliseconds left to reach an Earth year. This is naturally the same as we would get with Einstein's formula. Still, the new way of writing the formula gives additional insight.

Circular orbit's gravitational time dilation

The time dilation for a clock at circular orbit ${ }^{1}$ is given by

$$
t_{0}=t_{f} \sqrt{1-\frac{3}{2} \frac{2 G M}{r c^{2}}}=\sqrt{1-\frac{3}{2} \frac{v_{e}^{2}}{c^{2}}}
$$

where $v_{e}$ is the traditional escape velocity. We can rewrite this in the form of quantized escape velocity (derived above).

$$
\begin{gathered}
t_{o}=t_{f} \sqrt{1-\frac{3}{2} \frac{v_{e, p}^{2}}{c^{2}}} \\
t_{o}=t_{f} \sqrt{1-\frac{3}{2} \frac{\left(c \sqrt{\left.2 N \frac{l_{p}}{r}\right)^{2}}\right.}{c^{2}}} \\
t_{o}=t_{f} \sqrt{1-\frac{3 N l_{p}}{r}}
\end{gathered}
$$

\section{The Schwarzschild Radius}

The Schwarzschild radius [14] [15] of a mass $M$ is given by

$$
r_{s}=\frac{2 G M}{c^{2}}
$$

Rewritten into the quantum realm as described in this article, it must be

$$
\begin{gathered}
r_{s}=\frac{2 G_{p} M}{c^{2}} \\
r_{s}=\frac{2 G_{p} N m_{p}}{c^{2}} \\
r_{s}=\frac{2 \frac{l_{p}^{2} c^{3}}{\hbar} N \frac{\hbar}{l_{p}} \frac{1}{c}}{c^{2}}
\end{gathered}
$$

${ }^{1}$ At orbital radius larger then $\frac{3}{2} r_{s}$. 


$$
r_{s}=2 N l_{p}
$$

For a clock at the Schwarzschild radius, we get a time dilation of

$$
t_{o}=t_{f} \sqrt{1-\frac{2 N l_{p}}{2 N l_{p}}}=0 .
$$

At the Schwarzschild radius, time stands still. For a radius shorter than that the gravitational time dilation equation above breaks down. ${ }^{2}$

\section{Mass in Schwarzschild meters}

The Schwarzschild mass in terms of meters is given by

$$
\text { meter }=\frac{G M}{c^{2}}
$$

This can be rewritten as

$$
\begin{gathered}
\text { meter }=\frac{G_{p} N m_{p}}{c^{2}} \\
\text { meter }=\frac{\frac{l_{p}^{2} c^{3}}{\hbar} N \frac{\hbar}{l_{p}} \frac{1}{c}}{c^{2}} \\
\text { meter }=N l_{p}
\end{gathered}
$$

\section{Quantized Gravitational Bending of Light}

The angle of deflection in Einstein's General Relativity theory is given by

$$
\delta_{G R}=\frac{4 G M}{c^{2} r}
$$

This can be rewritten as

$$
\begin{gathered}
\delta_{G R H}=\frac{4 G_{p} M}{c^{2} r} \\
\delta_{G R H}=\frac{4 G_{p} N m_{p}}{c^{2} r} \\
\delta_{G R H}=\frac{4 \frac{l_{p}^{2} c^{3}}{\hbar} N \frac{\hbar}{l_{p}} \frac{1}{c}}{c^{2} r} \\
\delta_{G R H}=4 N \frac{l_{p}}{r}
\end{gathered}
$$

where $N$ is the number of Planck masses making up the mass we are interested in. From the formula above, this means that the deflection of angles comes in quanta. Lets also "control" that our Planck scale deflection rooted in Planck and GR is consistent for large bodies like the Sun, for example. The solar mass is $M_{s} \approx 1.988 \times 10^{30} \mathrm{~kg}$. The Sun's mass in terms of the number of Planck masses must be $\frac{1.988 \times 10^{30}}{2.17651 \times 10^{-8}} \approx 9.134 \times 10^{37}$. Further, the radius of the Sun is $r_{s} \approx 696342000$ meters. We can just plug this into the Planck scale deflection:

\footnotetext{
${ }^{2}$ Except if we assume the $l_{p}$ represents the radius of an indivisible particle. Thus if we move away from the point particle concept, this
} would simply mean that we could not go below the Planck scale Schwarzschild Radius. 


$$
\delta_{G R H}=\frac{4 N l_{p}}{r}=\frac{4 \times 9.134 \times 10^{37} \times 1.61622837 \times 10^{-35}}{696342000} \approx 8.48 \times 10^{-06}
$$

If we multiply this by $\frac{648000}{\pi}$, we get a bending of light of about 1.75 arcseconds or $\frac{1.75}{3600}$ of a degree. This is the same as has been confirmed by experiments and helped make Einstein famous, as Newton gravitation supposedly only predicted half of the bending of light. Newton's bending of light is given by

$$
\delta_{\text {Newton }}=\frac{2 G M}{c^{2} r}
$$

See for example [16] for derivations of bending of light under Newton's gravitation.

\section{Gravitational Redshift}

Einstein's gravitational redshift is given by

$$
\lim _{r \rightarrow+\infty} z(r)=\frac{1}{\sqrt{1-\frac{\frac{2 G M}{c^{2}}}{R_{e}}}}-1
$$

where $R_{e}$ is the distance between the center of the mass of the gravitating body and the point at which the photon is emitted. This we can rewrite as

$$
\begin{gathered}
\lim _{r \rightarrow+\infty} z(r)=\frac{1}{\sqrt{1-\frac{\frac{2 G M}{c^{2}}}{R_{e}}}}-1 \\
\lim _{r \rightarrow+\infty} z(r)=\frac{1}{\sqrt{1-\frac{1 \frac{l_{p}^{2} c^{3}}{\hbar} N \frac{\hbar}{l_{p}} \frac{1}{c}}{c^{2}}}}-1 \\
\lim _{r \rightarrow+\infty} z(r)=\frac{\frac{1}{\sqrt{1-\frac{2 N l_{p}}{R_{e}}}}-1}{\sqrt{1-\frac{R_{e}}{R_{g}}}}
\end{gathered}
$$

Further, in the Newtonian limit when $R_{e}$ is sufficiently large compared to the Schwarzschild radius, we can approximate the above expression with

$$
\begin{gathered}
\lim _{r \rightarrow+\infty} z(r) \approx \frac{G M}{c^{2} R_{e}} \\
\lim _{r \rightarrow+\infty} z(r) \approx \frac{\frac{l_{p}^{2} c^{3}}{\hbar} N \frac{\hbar}{l_{p}} \frac{1}{c}}{c^{2} R_{e}} \\
\lim _{r \rightarrow+\infty} z(r) \approx \frac{N l_{p}}{R_{e}}
\end{gathered}
$$

\section{Einstein's Field Equation}

And finally we get to Einstein's field equation. It is given by 
Table 1. The table shows some of the standard gravitational relationships given by Newton and Einstein and their expression in quantized form.

\begin{tabular}{|c|c|c|}
\hline Units & Newton and Einstein form & Quantized-form \\
\hline Gravitational constant & $G \approx 6.67408 \times 10^{-11}$ & $G_{p}=\frac{l_{p}^{2} c^{3}}{\hbar}$ \\
\hline Newton’s gravitational force & $F_{G}=G \frac{M_{1} M_{2}}{r^{2}}$ & $F_{G}=N_{1} N_{2} \frac{\hbar c}{r^{2}}$ \\
\hline Newton’s gravitational force & $F_{G}=G \frac{m_{p} m_{p}}{l_{p}^{2}}$ & $F_{G}=G_{p} \frac{m_{p} m_{p}}{r^{2}}=\frac{l_{p}^{2} c^{3}}{\hbar} \frac{\frac{\hbar}{l_{p}} \frac{1}{c} \frac{\hbar}{l_{p}} \frac{1}{c}}{l_{p}^{2}}=\frac{\hbar}{l_{p}} \frac{c}{l_{p}}$ \\
\hline Kepler's third law & $\frac{P^{2}}{a^{3}}=\frac{4 \pi}{G\left(M_{s}+m\right)}$ & $\frac{P^{2}}{a^{3}}=\frac{4 \pi}{l_{p} c^{2}\left(N_{1}+N_{2}\right)}$ \\
\hline Newton's escape velocity from any $n$ & $\nu_{e}=\sqrt{\frac{2 G M}{r}}$ & $v_{e, p}=c \sqrt{2 N \frac{l_{p}}{r}}$ \\
\hline Orbital velocity for any mass & $v_{o} \approx \sqrt{\frac{G M}{r}}$ & $v_{o, p} \approx c \sqrt{N \frac{l_{p}}{r}}$ \\
\hline Gravitational parameter & $\mu=G M$ & $\mu_{p}=N l_{p} c^{2}$. \\
\hline Gravitational acceleration field & $g=\frac{G M}{r^{2}}$ & $g_{p}=\frac{N l_{p} c^{2}}{r^{2}}$ \\
\hline Gravitational time dilation & $t_{0}=t_{f} \sqrt{1-\frac{2 G M}{r c^{2}}}=\sqrt{1-\frac{v_{e}^{2}}{c^{2}}}$ & $t_{o}=t_{f} \sqrt{1-2 N \frac{l_{p}}{r}}$ \\
\hline Orbital time dilation & $t_{0}=t_{f} \sqrt{1-3 \frac{G M}{r c^{2}}}=\sqrt{1-\frac{3}{2} \frac{v_{e}^{2}}{c^{2}}}$ & $t_{o}=t_{f} \sqrt{1-3 N \frac{l_{p}}{r}}$ \\
\hline Schwarzschild radius & $r_{s}=\frac{2 G M}{c^{2}}$ & $r_{s}=2 N l_{p}$ \\
\hline Newton bending of light & $\delta_{N}=\frac{2 G M}{c^{2} r}$ & $\delta_{N H}=\frac{2 N l_{p}}{r}$ \\
\hline Einstein bending of light & $\delta_{G R}=\frac{4 G M}{c^{2} r}$ & $\delta_{G R H}=\frac{4 N l_{p}}{r}$ \\
\hline Black holes & Possible & Depends on quantum interpretation \\
\hline Gravitational red-shift & $\lim _{r \rightarrow+\infty} z(r)=\frac{1}{\sqrt{1-\frac{\frac{2 G M}{c^{2}}}{R_{e}}}}-1$ & $\lim _{r \rightarrow+\infty} z(r)=\frac{1}{\sqrt{1-\frac{2 N l_{p}}{R_{e}}}}-1$ \\
\hline Gravitational red-shift approx & $\lim _{r \rightarrow+\infty} z(r) \approx \frac{G M}{c^{2} R_{e}}$ & $\lim _{r \rightarrow+\infty} z(r) \approx \frac{N l_{p}}{R_{e}}$ \\
\hline
\end{tabular}

I am far from an expert on Einstein's field equation, but based on the Planck gravitational constant given in this paper, we can rewrite it as

$$
R_{\mu v}-\frac{1}{2} g_{\mu v} R=\frac{8 \pi G_{p}}{c^{4}} T_{\mu v}
$$




$$
\begin{aligned}
& R_{\mu v}-\frac{1}{2} g_{\mu v} R=\frac{8 \pi \frac{l_{p}^{2} c^{3}}{\hbar}}{c^{4}} T_{\mu v} \\
& R_{\mu v}-\frac{1}{2} g_{\mu v} R=\frac{8 \pi l_{p}^{2}}{\hbar c} T_{\mu v}
\end{aligned}
$$

Bear in mind $\hbar=\frac{h}{2 \pi}$ and based on this we can alternatively write Einstein's field equation as

$$
R_{\mu v}-\frac{1}{2} g_{\mu v} R=\frac{16 \pi^{2} l_{p}^{2}}{h c} T_{\mu v}
$$

The potential interpretation and usefulness of this rewritten version of Einstein's field equation we leave to other experts for consideration. An interesting question is naturally whether or not it is consistent with some of the derivations given above in this form.

\section{Table Summary}

Table 1 summarizes our rewriting of some gravitational formulas. The output is still the same, but based on this view of gravity, masses, gravitational time dilation, and even escape velocity all come in discrete steps.

\section{Conclusion}

By making the gravitational constant be a function form of the reduced Planck constant, one can easily rewrite many of the end results from Newton and Einstein's gravitation in quantized form. Even if this is seen as an ad hoc method, it could still give new insight into what degree quantized Newton's gravitation and General Relativity are consistent with the quantum realm.

\section{Acknowledgements}

Thanks to Victoria Terces for helping me edit this manuscript. Also thanks to an anonymous referee for useful comments.

\section{References}

[1] Newton, I. (1686) Philosophiae Naturalis Principia Mathematica. Joseph Streater, London.

[2] Planck, M. (1901) Ueber das gesetz der energieverteilung im normalspectrum. Annalen der Physik, 309, 553-563. http://dx.doi.org/10.1002/andp.19013090310

[3] Bisnovatyi-Kogan, G.S. (2006) Checking the Variability of the Gravitational Constant with Binary Pulsars. International Journal of Modern Physics D, 15, 1047. http://dx.doi.org/10.1142/S0218271806008747

[4] Galli, S., Melchiorri, A., Smoot, G.F. and Zahn, O. (2009) From Cavendish to Planck: Constraining Newton’s Gravitational Constant with CMB Temperature and Polarization Anisotropy. Physical Review D, 80, Article ID: 023508. http://dx.doi.org/10.1103/physrevd.80.023508

[5] Fixler, B., Foster, G.T., McGuirk, J.M. and Kasevich, M.A. (2007) Atom Interferometer Measurement of the Newtonian Constant of Gravity. Science, 315, 74-77. http://dx.doi.org/10.1126/science.1135459

[6] Schlamminger, S. (2014) Fundamental Constant: A Cool Way to Measure Big G. Nature, 510, 478-480. http://dx.doi.org/10.1038/nature13507

[7] Rosi, G., Sorrentino, F., Cacciapuoti, L., Prevedelli, M. and Tino, G.M. (2014) Precision Measurement of the Newtonian Gravitational Constant Using Cold Atoms. Nature, 510, 518-521. http://dx.doi.org/10.1038/nature13433

[8] Ade, P.A.R., Aghanim, N., et al. (2015) Planck Intermediate Results. XXIV. Constraints on Variations in Fundamental Constants. Astronomy \& Astrophysics, 580, A22.

[9] Haug, E.G. (2016) The Gravitational Constant and the Planck’s Units: A Simplification of the Quantum Realm. http://vixra.org/pdf/1603.0207v5.pdf

[10] Schutz, B. (2003) Gravity from the Ground up. Cambridge University Press, Cambridge. http://dx.doi.org/10.1017/CBO9780511807800 
[11] Augousti, A.T. and Radosz, A. (2006) An Observation on the Congruence of the Escape Velocity in Classical Mechanics and General Relativity in a Schwarzschild Metric. European Journal of Physics, 376, 331-335. http://dx.doi.org/10.1088/0143-0807/27/2/015

[12] Tipler, P.A. and Llewellyn, R.A. (2012) Modern Physics. 6th Edition, W. H. Freeman and Company, New York.

[13] Einstein, A. (1916) Näherungsweise integration der feldgleichungen der gravitation. Sitzungsberichte der Königlich Preussischen Akademie der Wissenschaften, Berlin, 668-696.

[14] Schwarzschild, K. (1916) Üeber das gravitationsfeld einer kugel aus inkompressibler flussigkeit nach der einsteinschen theorie. Sitzungsberichte der Deutschen Akademie der Wissenschaften zu Berlin, Klasse fur Mathematik, Physik, und Technik, Berlin, 424.

[15] Schwarzschild, K. (1916) Über das gravitationsfeld eines massenpunktes nach der einsteinschen theorie. Sitzungsberichte der Deutschen Akademie der Wissenschaften zu Berlin, Klasse fur Mathematik, Physik, und Technik, Berlin, 189.

[16] Soares, D.S.L. (2009) Newtonian Gravitational Deflection of Light Revisited. https://arxiv.org/abs/physics/0508030 


\section{Appendix: Escape Velocity}

Derivation of the escape velocity from Planck scale

$$
\begin{gathered}
E \approx \frac{1}{2} m v^{2}-\frac{G m M}{r} \\
E \approx \frac{1}{2} N_{1} m_{p} v^{2}-\frac{G N_{1} m_{p} N_{2} m_{p}}{r} \\
E \approx \frac{1}{2} N_{1} \frac{\hbar}{l_{p}} \frac{1}{c} v^{2}-\frac{N_{1} \frac{l_{p}^{2} c^{3}}{\hbar} \frac{\hbar}{l_{p}} \frac{1}{c} N_{2} \frac{\hbar}{l_{p}} \frac{1}{c}}{r} \\
E \approx \frac{1}{2} N_{1} \frac{\hbar}{l_{p}} \frac{1}{c} v^{2}-N_{1} N_{2} \frac{\hbar}{r} c
\end{gathered}
$$

where $N_{1}$ is the number of Planck masses in the smaller mass $m$ (for example a rocket) and $N_{2}$ is the number of Planck masses in the other mass. This we have to set to 0 and solve with respect to $v$ to find the escape velocity:

$$
\begin{gathered}
\frac{1}{2} N_{1} \frac{\hbar}{l_{p}} \frac{1}{c} v^{2}-N_{1} N_{2} \frac{\hbar}{r} c=0 \\
v^{2}=2 \frac{N_{1} N_{2} \frac{\hbar}{r} c}{N_{1} \frac{\hbar}{l_{p}} \frac{1}{c}} \\
v^{2}=2 N_{2} \frac{l_{p} c^{2}}{r} \\
v=c \sqrt{2 N_{2} \frac{l_{p}}{r}}
\end{gathered}
$$

This is a quantized escape velocity. Since $N_{1}$ cancels out, we can simply call $N_{2}$ for $N$ and write the escape velocity as

$$
v=c \sqrt{2 N \frac{l_{p}}{r}}
$$

where $N$ is the number of Planck masses in the mass we are trying to escape from. 